\title{
Les méthodes d'extinction dans le Sud-Est
}

Avant de nous livrer à un inventaire et à un descriptif plus ou moins détaillé des techniques, il paraît nécessaire d'énoncer une idée générale, qui servira d'ailleurs de plan à l'exposé.

Comme Napoléon le disait de la guerre, on peut affirmer que l'extinction des incendies de forêts est un art simple, et tout d'exécution.

II faut et il suffit :

"Que l'on fasse parvenir à l'endroit voulu, au moment opportun, le matériel et le personnel nécessaires, et que celui-ci mène bien le combat. "

Nous allons reprendre cette formule, dans une première partie en exposant la doctrine et dans une seconde partie les réalités. Nous essaierons enfin de discerner les directions de l'évolution en cours.

\section{LA DOCTRINE}

On distingue plusieurs phases dans l'extinction des incendies de forêts : les deux principales sont la distribution des secours et l'emploi des moyens.

\section{- Distribution des secours}

C'est l'opération qui consiste à répartir sur le terrain, dans le cadre géographique du département, les moyens en personnel et matériel. La distribution des secours, vite et bien faite, conditionne pour une part importante le résultat final de l'opération. Cette phase débute au moment de l'alerte et se termine au moment où les secours arrivent sur place.

Pour qu'elle soit bien menée, plusieurs conditions doivent être réunies, qu'il s'agisse de la collecte des informations, de la décision, du départ des corps ou des itinéraires.

\section{Les informations}

Les décisions prises ne seront valables que dans la mesure où elles seront appuyées sur des informations précises et complètes. Ces informations sont de nature générale ou spécifique.

\section{Informations générales}

II s'agit de toutes celles qui ont trait à la situation du moment au niveau départemental, et elles sont de plusieurs sortes.

- Météorologie. Chaque département est divisé en plusieurs secteurs météorologiques. Pour chacun de ces secteurs, le Service météorologique régional diffuse deux fois par jour un bulletin d'information dont le commentaire technique se termine par une appréciation du risque de propagation, ainsi graduée : risque faible, habituel, sévère ou extrêmement sévère. Cette échelle tient compte essen- 
tiellement de la sécheresse du sol (bilan hydrique) et du vent prévu. De caractère assez synoptique, ces indications ont souvent besoin d'être affinées ou complétées par des mesures locales, les microclimats pouvant difficilement être analysés au niveau régional. C'est ce que font plusieurs départements, par des relevés pluriquotidiens suivant un réseau départemental plus ou moins dense.

Le bilan hydrique est diffusé deux fois par semaine pour chacun des secteurs météorologiques départementaux.

Le bilan hydrique situe sur une échelle de 0 à 150 le niveau de sécheresse du sol et par conséquent celui de l'eau de constitution des végétaux, en voie de décroissance tout au long de l'été du fait du ralentissement de la vie végétative. II est calculé par une formule mathématique intégrant en particulier les précipitations et l'évapo-transpiration des jours précédents.

Ces éléments météorologiques sont d'importance, car ils aident l'autorité responsable à se faire une idée assez juste du risque au moment du départ d'un feu. Avant même d'avoir des informations plus précises sur le comportement du feu lui-même et ses caractéristiques, il est ainsi possible au chef d'ajuster dès le départ les moyens au risque.

II nous manque encore une information précieuse, que pourra peut-être un jour nous donner la météorologie nationale, c'est une sorte d'indice d'éclosion, ou en d'autres termes une indication qui permette d'évaluer les dangers d'éclosion. Ceux-ci sont en effet souvent différents du risque de propagation qui est maintenant bien ajusté. Un tel indice permettrait aux services d'incendie de mettre en place en serrant de plus près la réalité les dispositifs prévisionnels d'opportunité prévus dans les plans départementaux. La météorologie nationale y travaille, et semble devoir approcher de résultats exploitables sur le plan opérationnel.

Enfin dans certains départements tout ou partie des guetteurs a suivi des cours spéciaux leur permettant d'effectuer des observations précieuses pour les P.C. Sans atteindre la rigueur des calculs effectués par les spécialistes de la météorologie, les observations ou même indications prévisionnelles de ces guetteurs sont souvent précieuses pour les P.C., aussi bien au niveau de la distribution des secours que de l'emploi des moyens.

- Moyens. Nous ne dirons rien d'original, car il est bien évident que la situation des moyens entre pour une part importante dans la décision du chef.

Tous les moyens, en personnel et en matériel, leur état de disponibilité, leur emplacement sur le terrain, doivent être disposés clairement sous les yeux du chef responsable. Dans les P.C. bien organisés, chacun des moyens, terrestres ou aériens, est figuré par une punaise magnétique bien diversifiée par sa forme, sa couleur et ses dimensions. Rangées au départ sur un tableau suivant une disposition claire et logique, très simple à lire, les punaises sont placées sur une carte au fur et à mesure des déplacements. II en est de même des unités isolées comme les Harkis ou des moyens aériens.

Simple en début de journée, l'information précise sur la situation des moyens devient de plus en plus difficile à tenir à jour au fur et à mesure que les départs et les déplacements se multiplient. Certains départements ont ainsi à suivre très souvent les déplacements de plusieurs dizaines d'engins. La moindre erreur peut avoir des effets importants sur le terrain. Compte tenu du fait que le réseau radio est très souvent saturé et que d'autre part les unités au combat sont particulièrement absorbées par leur mission immédiate, il arrive malheureusement trop souvent que des erreurs se produisent.

\section{Informations spécifiques}

Le responsable, nous l'avons vu, dispose d'un certain nombre d'informations générales qui sont un élément fondamental pour ses décisions. Ceci n'est pas suffisant. II lui faut encore dəs informations particulières pour chacun des feux.

- Situation. Pour l'acheminement des secours, un renseignement approximatif lui suffit, car il peut être affiné par la suite, au moment où les secours approchent du feu.

Par contre il lui faut situer exactement le feu sur une carte à grande échelle bien renseignée pour apprécier convenablement le risque. Cet élément est important dans la décision qu'il doit prendre très vite sur la détermination des moyens à envoyer. La situation précise du feu sur une carte va lui permettre d'apprécier très vite les risques dus au terrain - relief, éventuellement accès, pare-feu naturels ou construits, etc... Joints aux éléments météorologiques connus, ces informations vont peser d'un grand poids dans le choix des moyens à faire partir.

- Évolution. Au niveau de la distribution des secours, le chef ne doit pas attendre qu'on le lui demande pour faire partir des renforts. L'un de ses soucis doit être de réunir le plus possible d'informations sur l'évolution du feu, avant même de recevoir les premiers messages des secours arrivés sur place. Les guetteurs bien instruits lui seront précieux. Ils sauront lui parler de la vitesse de propagation, lui donneront par l'observation des fumées des indications intéressantes sur le vent local. Et puis le chef s'efforcera d'obtenir des renseignements complémentaires par tous les moyens possibles, en téléphonant au maire, au gendarme, aux voisins s'il le peut, en envoyant rapidement sur place une reconnaissance terrestre ou aérienne. 
Au cours même des opérations d'extinction il est en principe mieux renseigné par le commandant au feu, mais il arrive souvent que lui-même puisse, grâce à ses moyens d'observation, donner des indications utiles sur la conduite des opérations, restructurer le commandement sur le terrain, ou faire partir des renforts nouveaux avant qu'on ne les lui demande.

\section{La décision}

Nous venons de passer rapidement en revue les bases à partir desquelles seront prises les décisions, à différents niveaux, et ce qu'elles seront.

- Niveau communal. Deux préoccupations principales. La première est de faire partir les premiers secours, surtout si le maire dispose d'un corps de sapeurs-pompiers dans sa commune, et la seconde est de pousser le plus d'informations possible sur le P.C. départemental. Dans certains départements il existe des commissions communales des forêts, créées pour satisfaire aux besoins de la lutte dans son ensemble, qui ont justement pour mission de recueillir les informations, de guider les secours, de conseiller le commandant au feu et d'assurer des missions de logistique locale en matière de vivres et d'essence.

De toute façon c'est à ce niveau que seront collectées les informations spécifiques sur le feu déclaré, permettant d'ajuster au mieux l'importance des secours à faire partir.

- Niveau départemental. Rôle du P.C. fixe du Service départemental d'incendie et de secours. Il faut voir juste. Dans le doute, faire partir trop de moyens plutôt que pas assez.

Le P.C. a la charge, et cela entre dans la distribution des secours, d'organiser en permanence le trafic radio en fonction de l'évolution des situations et de mettre en place les dispositifs de guet, d'alerte et d'intervention, avant tout départ d'incendie, en fonction de la situation météorologique, en exécution des plans départementaux de mobilisation progressive.

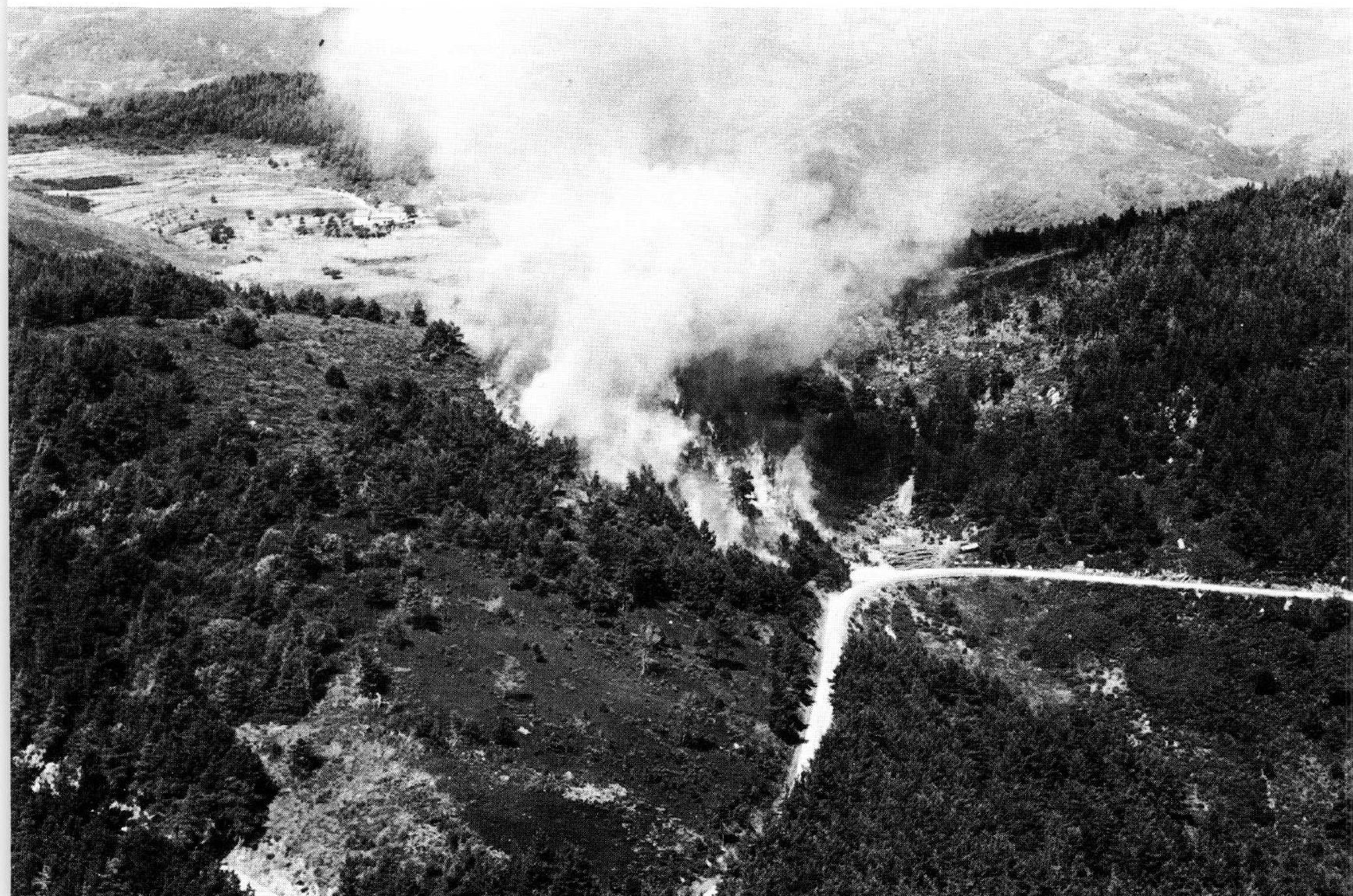


Au moment du feu, il a la charge de décider des moyens nécessaires. Ceci ne veut pas dire que l'on doive obligatoirement passer par lui pour le départ des premiers secours. Si l'alerte est donnée par une tour de guet sur le réseau radio, c'est évidemment le P.C. qui donne les premiers ordres. Si l'alerte est donnée par un témoin, elle aboutira le plus souvent à la gendarmerie locale ou à la mairie qui feront partir les premiers secours locaux. Les structures départementales varient d'ailleurs sensiblement dans ce domaine. Certains départements possèdent un P.C. départemental bien organisé et une structuration très centralisée. D'autres, moins menacés par les incendies de forêts, ont conservé les méthodes traditionnelles de distribution des secours, avec demandes de renforts émanant uniquement du commandant au feu. Entre ces deux extrêmes, plusieurs solutions mixtes sont adoptées, chaque département essayant d'adapter au mieux ses structures à ses risques.

Quelles que soient les méthodes, la distribution des secours doit être proportionnelle à l'importance du feu ou des feux. La décision doit porter sur tous les moyens, en les harmonisant pour obtenir un ensemble cohérent, bien adapté au risque et au terrain. Moyens terrestres des corps de sapeurs-pompiers, Harkis, Canadairs, reconnaissances terrestres et aériennes, fréquences radio à utiliser, désignation du commandant au feu.

- Départs et itinéraires. Les départs s'effectuent toujours sous le contrôle du P.C. départemental. En principe la quasi totalité des unités, sapeurs-pompiers ou autres est dotée de moyens radio. Dès le départ, liaison est prise avec le P.C., et l'heure est notée. II est alors relativement facile au P.C., compte tenu de I'heure et de la distance, de prévoir à peu près I'heure d'arrivée. Ce renseignement est aussitôt transmis au commandant au feu, et il lui sera très utile pour la conception de son idée de manœuvre. Toujours dans le même cadre, c'est un échange de vues entre le commandant au feu et le P.C. qui permettra d'arrêter les itinéraires à suivre par les différentes unités. Sur un feu important en particulier les itinéraires pourront varier selon les unités, pour qu'elles arrivent avec le moins de perte de temps possible au point qui leur est assigné dans le dispositif prévu. Le guidage des unités sur les itinéraires est une mission qui entre dans la distribution des secours et revient au P.C. départemental, au besoin sur une fréquence radio distincte de la fréquence opérationnelle pour décharger le réseau.

Enfin, pendant ces mouvements d'acheminement, il appartient au P.C. départemental de préciser les coordonnées. Celles-ci manquent quelquefois de précision au départ, et sont données quelquefois sous forme de coordonnées polaires, ou de lieu-dit, ou de points de concentration des renforts avant acheminement définitif. Dès que le P.C. a pu par les moyens indiqués plus haut affiner ses informations, il les transmet aux unités en transit sous forme de coordonnées de chasse.

\section{- Emploi des moyens}

Tous ces moyens, convergeant le plus vite possible vers l'incendie, doivent être pris en charge pour l'action par un organe coordinateur. C'est le rôle du P.C. opérations, outils de travail du commandant au feu. Nous allons examiner ses conditions de travail, ses missions et son action.

\section{Conditions de travail}

II ne sera pas traité ici du petit feu, ne dépassant pas quelques hectares et ne présentant pas de menace grave dans des conditions météorologiques sévères. II est traité rapidement, par une attaque en pointe sans grande difficulté.

Nous ne traiterons que du feu dangereux. Le moins qu'on puisse dire des conditions de travail c'est qu'elles sont très difficiles. Le P.C. est constitué, au départ, par la voiture de liaison radio du premier chef de corps arrivé sur les lieux, souvent seul avec un conducteur. C'est à cet homme seul qu'il appartient d'appliquer la formule indiquée au début de ce texte, c'est-à-dire déterminer les points d'attaque en fonction de l'évolution du feu à prévoir pendant le transit des secours, apprécier rapidement les moyens dont il aura besoin, et arrêter les tactiques à employer.

Pour faire tout cela, il ne dispose que des quelques informations qu'il a pu glaner à l'écoute radio pendant la route, avec des difficultés pour prendre des notes.

Petit à petit les moyens arrivent, et dans les meilleures circonstances le P.C. commence à s'étoffer en personnel et quelquefois en matériel, permettant ainsi au chef de se soustraire aux tâches mineures d'exploitation radio, de liaison, de secrétariat indispensable à la poursuite des opérations. Le plus généralement, le P.C. n'est à peu près installé qu'à la fin de la phase de mise en place. Jusque-là le chef est harcelé; il a tout à faire, s'informer, prévoir, reconnaître, guider, ordonner, contrôler et prendre note des mouvements s'il veut s'y retrouver au bout d'un moment.

Il est très mal outillé pour le faire, et il est difficile de faire autrement avec le niveau d'équipement qui paraît être admis partout à l'heure actuelle. 


\section{Les missions}

Alors que le P.C. fixe départemental fait surtout de la distribution des secours, le P.C. opérationnel, organe de commandement du commandant au feu, fait à la fois de la distribution des secours en répartissant les moyens sur l'ensemble de la ligne de feu, et de l'emploi en donnant des missions précises aux chefs de secteurs et chef de corps. Les missions qu'il peut donner sont de plusieurs sortes. Elles correspondent en fait aux différents emplois tactiques qui peuvent être faits du personnel et des matériels. Nous allons examiner successivement les différents procédés de combat.

\section{Techniques}

L'eau d'abord, sous toutes ses formes. Depuis la lance à orifice de $18 \mathrm{~mm}$ alimentée par une pompe de 30 ou $60 \mathrm{~m}^{3} / \mathrm{h}$, rarement utilisée parce qu'elle consomme trop d'eau, jusqu'au seau pompe dorsal à pompe à main avec à la lance un orifice de 2 à $3 \mathrm{~mm}$. La lance la plus employée est celle de $7 \mathrm{~mm}$, alimentée par des tuyaux à parois internes lisses de $25 \mathrm{~mm}$ avec pompe de 6 à $30 \mathrm{~m}^{3} / \mathrm{h}$. Matériel léger, qui autorise de longs établissements en terrain difficile. Les pertes de charge sont cependant importantes, et nécessitent souvent la mise en place de relais avec les motopompes portatives, qui ne pèsent que $25 \mathrm{~kg}$. Les avions CL 215 Canadairs peuvent effectuer sur les feux des largages importants, grâce à leur citerne de $6 \mathrm{~m}^{3}$. Enfin une technique originale a été mise au point sous le nom de commandos héliportés. Elle permet de transporter sur les fronts inaccessibles des personnels spécialisés, pompes et tuyaux légers, et de ravitailler le commando en eau à l'aide de citernes souples de 800 I transportées en cargo-sling par les hélicoptères. La descente des personnels se fait au cordage quand le poser n'est pas possible. Cette technique permet d'atteindre n'importe quel point inacessible autrement. Malheureusement les prix de revient sont trop élevés pour les collectivités locales, et cette technique, qui a fait ses preuves, ne pourra être utilisée que quand les armées consentiront à ne pas envoyer la facture aux utilisateurs.

A l'eau peuvent être ajoutés des produits chimiques pour augmenter son efficacité. Produits dits « mouillants » d'abord, facilitant la pénétration par abaissement de la tension superficielle : diminuent le ruissellement, évitent le gaspillage, éteignent plus vite. Produits "retardants " à plus ou moins longue rémanence, effet contraire des mouillants, utilisés pour établir des lignes d'arrêt en avant du front de feu.

Autre technique couramment utilisée, le battage des flammes. On a semble-t-il abandonné les battes à feux, d'un rendement médiocre et d'une utilisation pénible, pour les remplacer par la classique branche verte coupée à la serpe. Pour utiliser cette technique, il faut être au contact des flammes, ce qui interdit son emploi sur les points,à haut potentiel calorifique.

Enfin la technique ancestrale du contre-feu. Faire table rase en avant du front de feu. Procédé très efficace, mais difficile et dangereux. De plus, les résultats sont très aléatoires si le contre-feu est mal conduit. Sans nous étendre trop longtemps sur cette technique, bien connue des lecteurs de la Revue Forestière, il nous faut bien dire qu'elle n'est pas abandonnée. A notre sens, des efforts doivent être faits, dans le domaine de l'instruction, pour que le contre-feu soit convenablement pratiqué. Ultime ressource, il ne doit être ni négligé ni pratiqué couramment. II reste cependant le dernier moyen à notre disposition pour arrêter les pointes d'un grand feu quand tout le reste a échoué ou s'avère impossible.

On peut encore citer, à cheval entre la prévention, la prévision et l'extinction, la tranchée pare-feu tactique effectuée à la hâte pour appuyer une ligne d'arrêt ou un contre-feu. Construite aux outils à main ou au moyen d'engins, elle est très utile et permet parfois de conduire une action efficace impossible autrement.

\section{Tactiques}

On a souvent déploré, dans des milieux mal informés, qu'il n'existe pas un plan d'intervention. Mot magique. Le plan résoud tous les problèmes. On s'étonne de voir sur le terrain des actions improvisées, et les observateurs extérieurs constatent un certain désordre, plus ou moins grand, à partir de leur point d'observation, qu'il s'agisse d'une observation optique d'un bord de route ou acoustique près d'un émetteur-récepteur radio. II faut cependant percer la surface et aller plus avant, vers le fond des choses.

De quoi s'agit-il? De commander une opération, sur un terrain plus ou moins bien connu, contre un adversaire dangereux en situation offensive. Chaque département a son plan de lutte contre les incendies de forêts. II s'agit d'un document qui est à la fois un inventaire des moyens et des consignes de mise en œuvre en fonction des circonstances. Le plan de lutte concerne surtout le P.C. fixe départemental ; pour le P.C. opérations, c'est une affaire de commandement, pas une affaire de plan. Le commandant au feu n'applique pas et n'élabore pas un plan, essentiellement statique. II élabore une manœuvre, en suivant dans la mesure du possible l'ordre logique habituel : collecte et interprétation des informations, formulation de l'idée de manœuvre, mise en forme et émission des ordres, contrôle. Cette dernière opération conduira souvent, si les résultats ne concordent pas avec l'objectif fixé, à une reconsidération de l'idée de manœuvre. C'est là la façon logique, normale et efficace de raisonner et de travailler. Pas de plan préétabli, mais une technique de raisonnement tactique qui aboutisse à des solutions 
réalistes, applicables et suffisamment souples pour s'adapter au terrain et à l'évolution souvent très rapide de la situation. C'est un combat qu'on mène, avec ses aléas, ses victoires et ses défaites, et les erreurs que l'on commet, petites ou grandes, pour des raisons variables et malheureusement toujours très nombreuses.

II faudrait un volume pour étudier dans le détail les différentes possibilités de manœuvre qui s'offrent au chef. Nous allons examiner rapidement les plus importantes.

- Attaque de front. A l'eau simple ou avec mouillants. But : abattre les flammes, stopper la progression pour permettre ensuite l'extinction. Contraintes : personnel courageux et entraîné, moyens en eau puissants. Le diamètre des lances diminue au fur et à mesure que la diminution de la chaleur rayonnante permet d'approcher plus près. Risque de débordement par les ailes. Les avions-citernes sont d'un grand secours pour faciliter l'approche, en attaque directe des foyers.

- Attaque de flanc. A l'eau et mouillants ou par battage. But : extinction. L'approche est plus facile. Personnel moins spécialisé et en plus grand nombre pour pouvoir mener l'extinction totale à son terme. Progression échelonnée le long du flanc, avec équipe de pointe à l'eau, les autres avec outillage individuel.

- Percée de flanc. L'attaque sous le vent du front de feu étant parfois impossible et l'attaque de flanc insuffisante, on essaie d'attaquer le front par derrière, en passant dans le brûlé. La manœuvre consiste à ouvrir dans le flanc de feu un passage pour le personnel et le matériel, et à rattraper le front de feu par l'arrière. Le danger est moindre que par une attaque frontale. L'opération est intéressante, car elle diminue l'intensité du dégagement calorifique général et permet ensuite l'attaque de front. Elle est surtout pratiquée pour diminuer la température des courants de convection émis par un front de grande profondeur. Assez rarement pratiquée, car elle est très pénible pour le personnel et éprouvante pour le matériel du fait de la progression conduite sur un sol chaud et en pleine fumée.

- Ligne d'arrêt. Si les attaques directes s'avèrent impossibles, en particulier à cause des difficultés d'accès, la tactique utilisée est celle de la ligne d'arrêt, établie en avant du front de feu. Le but de la manœuvre est l'arrêt de la progression, suivie de l'extinction.

Elle est constituée par des personnels et des matériels que l'on met en place le long d'une piste ou d'une route. Le choix du terrain est à considérer de près, et tous les moyens possibles sont utilisés pour ralentir le front avant son arrivée sur la ligne d'arrêt : retardants par moyens aériens ou terrestres, débroussaillement rapide, voire petits contre-feux.

Cette tactique ne doit être utilisée que quand les attaques directes ont échoué ou n'ont pu être réalisées.

Dans la plupart des cas, en particulier sur un feu qui a pris de l'importance, on se trouve en présence de plusieurs fronts de feux, le front initial s'étant agrandi et fractionné en plusieurs pointes. Le souci du commandant au feu est alors d'utiliser au mieux les techniques et les tactiques décrites très sommairement ci-dessus, en fonction du terrain, de l'évolution du feu, et des moyens dont il dispose. On doit cependant considérer que la ligne d'arrêt est un pis-aller, et qu'elle doit le plus possible être précédée d'attaques directes, de front ou de flanc, qui même si elles n'emportent pas la décision sont susceptibles de ralentir l'avance du feu, de fractionner son front, et de diminuer son intensité.

\section{Organisation}

A partir d'une certaine importance, il est évident que le commandant au feu ne peut plus assurer directement son commandement.

Il faut alors s'organiser, et diviser l'ensemble en un certain nombre de secteurs, chacun d'eux étant placé sous le commandement d'un chef de secteur.

L'emploi des moyens passe alors pour la plus grande part aux chefs de secteurs, le commandant au feu s'attachant à distribuer judicieusement les secours dont il dispose entre les différents fronts.

L'articulation du réseau radio, en réseau libre ou dirigé, reste sous la responsabilité du commandant au feu, sur les fréquences qui lui sont indiquées par le P.C. fixe départemental. Chaque département a en effet accès à cinq fréquences générales, en plus des fréquences urbaines attribuées aux grandes villes. Quatre fréquences sont communes : intercommunication, dégagement, air/sol et commandement. La cinquième fréquence est propre à chacun des départements, pour éviter des interférences, c'est la fréquence opérationnelle départementale. Les liaisons doivent être établies d'une part entre les unités engagées et le commandant au feu, généralement sur la fréquence opérationnelle, et d'autre part entre le commandant au feu et le P.C. fixe départemental, généralement sur la fréquence de commandement. Compte tenu des reliefs souvent très accusés des régions méditerranéennes, les liaisons directes sont parfois difficiles. II convient alors d'utiliser les relais radio, soit par l'intermédiaire des points hauts fixes, soit par l'installation de relais d'opportunité, sous la forme d'un mobile radio mis en place sur une hauteur convenablement choisie. 
Le choix de l'emplacement du P.C. du commandant au feu est très important. II est soumis à un certain nombre de contraintes : possibilités d'observation, facilité des liaisons radio, accès facile, proximité des différents secteurs, éloignement relatif du front pour éviter des déplacements trop fréquents. En effet, le P.C. opérationnel doit être relativement fixe, bien signalé et sa position doit toujours être connue de l'ensemble des participants à l'opération. Le détachement d'un hélicoptère léger auprès du P.C. facilite considérablement son action dans tous les domaines, sur les plans de la collecte des informations, de la recherche des accès, du guidage des moyens terrestres et aériens, de la reconnaissance du risque par les chefs de secteur. Utilisé comme P.C. volant pour la conduite d'une opération déterminée, il permet de gagner du temps et d'obtenir une coordination des efforts dans l'espace et dans le temps souvent très difficile à réaliser sans son aide, voire impossible.

\section{LA RÉALITÉ}

\section{- Situation actuelle}

Ce qui vient d'être dit est un résumé des conceptions actuellement admises pour l'extinction des incendies de forêts. Il nous faut examiner, pour essayer d'être complet, dans quelle mesure ces conceptions sont appliquées sur le terrain. On peut répondre que les réalisations sont très variables. Les structures et les matériels varient en effet suivant les départements. Tous n'ont pas les mêmes risques ni la même organisation. Certains, bien pourvus sur le plan des moyens d'intervention, sont pauvres en moyens de commandement. D'autres manquent au contraire de matériel, ou de personnel d'exécution, ou d'instruction. On constate cependant, depuis quelques années, une meilleure prise de conscience des problèmes et une nette amélioration des dispositifs de lutte. De gros progrès sont encore à faire, dans plusieurs directions.

Recherches techniques d'abord, sur le plan d'une amélioration et d'une meilleure adaptation des matériels d'extinction. Elles sont conduites par plusieurs organismes, au niveau de l'Entente interdépartementale pour la lutte contre les incendies de forêts, du Centre technique du Génie rural, des eaux et des forêts, des services départementaux d'incendie, et des constructeurs.

Recherches tactiques et stratégiques, menées par les officiers de sapeurs-pompiers. De plus en plus on voit se généraliser les tactiques d'attaque directe, en utilisant les techniques décrites plus haut, et les établissements de tuyaux avec relais sur plusieurs centaines de mètres deviennent par exemple habituels dans certains départements. L'instruction des personnels, bien entreprise, doit à notre avis être poursuivie avec vigueur.

Création de moyens d'intervention, dont le financement repose en majeure partie sur les collectivités locales. Le point le plus faible semble se situer au niveau des moyens radio-téléphoniques, fixes et mobiles. Certains départements sont encore sous-équipés.

Moyens de commandement enfin, aussi bien au plan des état-majors, chargés des missions de conception, de coordination et de distribution des secours au niveau départemental, qu'au plan des moyens de commandement au niveau opérationnel. Cette observation est valable aussi bien pour les personnels, officiers et sous-officiers, que pour les matériels, installation des P.C. fixes et mobiles. C'est à notre sens le point principal, sur lequel il conviendrait de concentrer les efforts dans les années à venir.

Certains pensent, avec raison semble-t-il, qu'une amélioration considérable des résultats pourra être obtenue dans un proche avenir par une meilleure coordination des moyens existants. Une telle amélioration passe obligatoirement par un perfectionnement très sensible des moyens de commandement, qui sont encore nettement insuffisants, mais dont on commence à comprendre la nécessité à tous les niveaux de décision.

Colonel André HOUSCASTAGNÉ Inspecteur départemental

SERVICE DÉPARTEMENTAL

D'INCENDIE ET DE SECOURS

Centre Jacques Vion

Quartier Le Fournas

83015 DRAGUIGNAN 